\title{
Adaptive Automation of Human-Machine System Information-Processing Functions
}

\author{
David B. Kaber, North Carolina State University, Raleigh, North Carolina, Melanie C. \\ Wright, Duke University Medical Center, Durham, North Carolina, Lawrence J. Prinzel \\ III, NASA Langley Research Center, Hampton Roads, Virginia, and Michael P. Clamann, \\ Micro Analysis \& Design, Boulder, Colorado
}

\begin{abstract}
The goal of this research was to describe the ability of human operators to interact with adaptive automation (AA) applied to various stages of complex systems information processing, defined in a model of human-automation interaction. Forty participants operated a simulation of an air traffic control task. Automated assistance was adaptively applied to information acquisition, information analysis, decision making, and action implementation aspects of the task based on operator workload states, which were measured using a secondary task. The differential effects of the forms of automation were determined and compared with a manual control condition. Results of two 20-min trials of AA or manual control revealed a significant effect of the type of automation on performance, particularly during manual control periods as part of the adaptive conditions. Humans appear to better adapt to AA applied to sensory and psychomotor information-processing functions (action implementation) than to AA applied to cognitive functions (information analysis and decision making), and AA is superior to completely manual control. Potential applications of this research include the design of automation to support air traffic controller information processing.
\end{abstract}

\section{INTRODUCTION}

Recent research on human-automation interaction theory (Endsley \& Kaber, 1999; Parasuraman, Wickens, \& Sheridan, 2000) has sought to develop theoretical frameworks for making decisions about what complex system functions to automate, and to what extent, in advance of implementation and as part of the systems design process. This work has relevance to adaptive automation. Adaptive automation (AA) has been defined as the dynamic allocation of control of system functions to a human operator and/or computer over time with the purpose of optimizing overall system performance (see Kaber \& Riley, 1999; Parasuraman, 1987; Rouse, 1977; Scerbo, 1996). Dynamic control, or function, allocations are based on operator states and/or task contextual information.

Research on AA has focused on the perfor- mance and workload implications of allocations of manual and automated control of monitoring and psychomotor functions (e.g., tracking) during complex system operations. Several studies have demonstrated that AA improves performance in monitoring tasks in comparison with the use of static (fixed) automation under multitask circumstances (Hilburn, Molloy, Wong, \& Parasuraman, 1993; Parasuraman, Mouloua, Molloy, \& Hilburn, 1993). Unfortunately, little work has been conducted to examine the human performance implications of AA of system functions requiring cognitive aspects of information processing (e.g., Hilburn, Jorna, Byrne, \& Parasuraman, 1997). Reviews of AA research (Kaber, Riley, Tan, \& Endsley, 2001; Parasuraman, Mouloua, \& Molloy, 1996; Scerbo, 1996) have pointed to the paucity of empirical studies on AA for cognitive task performance. Beyond this, there is currently a need for research to make a 
systematic comparison of the performance effects of AA applied to various stages of information processing (Parasuraman, 2000; Parasuraman et al., 2000), including decision-making tasks. Models of types and levels of automation can be used to classify forms of AA and to identify general functions to which AA can be applied most successfully.

Building on earlier research on humancentered automation (Sheridan \& Verplanck, 1978; Wickens, Mavor, Parasuraman, \& McGee, 1998), Parasuraman et al. (2000) formulated a model-based approach to automation of complex systems by considering existing theories of human information processing (e.g., Wickens, 1992). Their motivation was that automation is not an "all-or-none" concept but may be defined in terms of type, or the stage of information processing to which automation is applied, and may occur at different levels. Four stages of information processing are represented in their model information acquisition, information analysis, decision making, and action implementation - and the level of automation of each stage may vary and defines, in part, the overall degree of system autonomy. As can be seen in many real-world systems, Parasuraman (2000) observed that automation could be applied to one stage of information processing or to multiple stages in combination. Although the authors conceptualized automation as a continuum and established that there may be unique ranges of automation for different functions, they also proposed a more general classification for types of automation in terms of five levels (none, low, medium, high, and full). The objective of the Parasuraman et al. (2000) work was to provide a framework for making automation design decisions on the basis of research on human performance costs of automation and empirical evaluation of various combinations of types and levels of automation.

The model of types and levels of automation developed by Parasuraman et al. (2000) was used as a basis for the current AA research, specifically in defining experimental conditions. We sought to gain insight into the performance and workload effects of the various forms of automation defined in the literature and to understand the implications of adaptively applying them to lower order versus higher order informationprocessing (IP) functions. (By high-order IP functions, we mean data integration and analysis, planning, and decision making relative to goals. By low-order IP functions, we mean stages that primarily support psychomotor performance, including information search/acquisition [perception] and response execution [motor control] relative to decision making.) We assessed the performance of a complex humanmachine system under AA, applied exclusively to each of the four stages of information processing included in the Parasuraman et al. (2000) model. The overall goal was to establish the differential effects of AA on cognitive and psychomotor task performance. To our knowledge, AA has not been previously investigated in this way. The advantage of this approach is that the automation framework provides a clear and concise basis within which to explain results and may facilitate comparison of findings across future studies.

The study was conducted using a low-fidelity simulation of air traffic control (ATC). Advanced automation is currently being applied to ATC to address large increases in the overall volume of traffic expected in the near future (Laois \& Giannacourou, 1995). However, the forms of ATC automation that have been developed (e.g., aircraft conflict detection aids, trajectory projection aids, and clearance advisory aids) attempt to address the planning and decision-making aspects of traffic management. Existing expert systems may have limitations that restrict complex information integration and critical decision making, which are required in ATC for effective performance (Leroux, 1993). In addition, a major human factors concern with these forms of high-order (planning and decision) automation is that they will lead to complacency and out-of-the-loop performance problems, including the loss of situation awareness (SA). Adaptive automation may be a better approach than the existing forms of advanced ATC automation in that it attempts to apply automation based on some consideration of human performance and/ or operator state. It may be possible to more effectively draw out benefits of high-order ATC automation by using an AA approach to implementation than would be possible with the current ATC automation concepts. Through analytical evaluation, Parasuraman (2000) suggested that ATC could bear high-level information 
acquisition and analysis automation and moderate decision-making and action implementation automation. Our investigation focused on adaptive allocation of those forms of automation to be implemented in ATC in order to further expand the empirical research basis for automation design decisions.

\section{Hypotheses}

On the basis of the prior AA research on psychomotor tasks (Parasuraman et al., 1993), we projected that AA of the lower order IP functions, including information acquisition and action implementation, would provide some benefit over completely manual control in the ATC simulation. We also expected that AA of higher order IP functions, including information analysis and decision selection, would be of some benefit to operator performance in comparison with a manual control condition, but not to the extent of AA of information acquisition and action implementation. Hilburn et al. (1997) found that AA of the decision function in a high-fidelity ATC simulation served to moderate controller mental workload better than did completely manual control or the use of static automation under variable traffic conditions. However, Endsley and Kaber (1999), in studying the effects of various forms of automation on dynamic control task performance, found that under normal system operating conditions, low-level, psychomotor automation yielded better performance than did higher level decision support or supervisory control automation. In their study, automation failures occurred at periodic intervals and operators were required to use manual control. Subsequent to a failure, the automation switched back "on.” Although the control mode switches were presented as system failures to the participants, the effect of AA on performance may be similar to that of system automation that shuts down when it fails.

Other research on cycles of automation in complex systems control (Hadley, Prinzel, Freeman, \& Mikulka, 1999) has demonstrated that in general, reversions to manual control after automation can have a negative effect on subsequent performance and appropriate cognitive resource allocation. Hadley et al. (1999) also found that there are greater "return-to-manual deficits" for shorter cycle automation. They ob- served that participants found it more difficult to reorient to, and sustain, manual control of a compensatory tracking task as the duration of a preceding automated control period decreased. (A P300 evoked response potential was significantly smaller for shorter cycles, indicating reduced perception of the mode transition and new IP needs.) There was also a significant increase in workload for shorter cycle automation. This study addressed this problem only for a psychomotor form of automation.

Based on this research, we suspected that any potential performance costs of AA applied to higher order IP functions in our task might be attributable to the degree of cognitive complexity of the automation functions and the requirement to switch between automated and manual control modes during AA trials - that is, the return-to-manual deficit problem (Hadley et al., 1999). By cognitive complexity, we mean the complexity of the user's mental model of interaction with the automation based on the number of contexts for which the automation is useful and the number of functions or operations that it performs (see Pervin, 1984; Rauterberg, 1992). We also speculated that the return-to-manual deficit problem would be worse when reverting to manual control from higher level automation (decision making). Given the cognitive complexity of information analysis and decision-making aids for ATC tasks, we expected that it would be more difficult for operators to disengage from the use of such tools, reorient, and return to manual control as compared with shifting from the use of data gathering or interface action support tools to manual control. Consequently, performance under AA of information analysis and decision making might suffer compared with returns to manual control when using information acquisition or action implementation aids. This expectation was also based, in part, on historical psychology research (e.g., water jug experiments) demonstrating human preference for the use of rules and heuristics in problem-solving tasks rather than using higher order cognitive processing, including decision-making skills (Atwood \& Polson, 1976).

With respect to task workload, we speculated that application of AA to the decision-making function would not be as effective as AA of the monitoring/information acquisition and action 
implementation aspects for managing operator load. Automation of aircraft data gathering and clearance execution was expected to reduce operator interface actions under high traffic complexity conditions and to have a direct effect on operator workload states. Automation of information integration and analysis (projection of potential trajectory conflicts), in contrast, was expected to reduce operator planning and selection efforts over time, and the implication of dynamic control allocations on operator load would not be as direct. Because of the potential return-to-manual deficit problem, and the relationship of the forms of automation to workload, we expected the action implementation mode to show the greatest performance benefit of AA.

\section{METHODOLOGY}

\section{Participants and Tasks}

Forty-seven North Carolina State University students were recruited for the experiment. All participants were required to have $20 / 20$ or corrected-to-normal visual acuity and personal computer experience. Seven of the participants were used in a pilot study to establish criterion levels for various dependent measures recorded during the actual experiment.

Two computer-based tasks - the ATC simulation (Multitask $^{\odot}$ 2.0; Kaber, Clamann, Labiad, \& Wright, 2001) and a secondary gauge-monitoring task - were used in this study. Both of the tasks were modified versions of the tasks used by Kaber and Endsley (1997) and Kaber and Riley (1999). In the present experiment, the secondary task provided a measure of primary task workload that was used as a basis for computermandated control allocations (manual or automated control of primary task functions). There was no advance warning of the dynamic function allocations (DFAs) provided to operators. They were instructed to allocate their attention to the primary, dynamic control task and to the secondary task to the extent they could.

The Multitask ${ }^{\odot}$ simulation presented participants with a radarscope revealing the position of different types of aircraft in a simulated airspace. Three different types of icons were used to graphically represent aircraft (military, commercial, and private). Aircraft initially appeared on the periphery of the scope and traveled to- ward a "home base" at the center of the display. The speed of an aircraft was dependent upon its type, and all aircraft required between 60 and $120 \mathrm{~s}$ to reach the base after their initial appearance on the scope. Up to six targets were presented on the radarscope at any given time, and this workload varied by only one target during each minute of the task.

The participant's task was to locate and "clear" aircraft for landing before they reached the home base or collided with another aircraft. Clearing an aircraft required two steps: establishing a communication link and issuing a clearance. Participants pointed to the aircraft with a mouse controller and pressed the left mouse button to establish a communication link. After a few seconds, participants issued a clearance by again pointing to the aircraft with the mouse and pressing the right button. A clearance was typically based on participant consideration of the type of aircraft, its location relative to the base and other aircraft, and its speed and stage of processing (e.g., communication link established, already cleared). When performed correctly, the entire task required $30 \mathrm{~s}$, and participants could clear multiple aircraft simultaneously. The steps as part of the clearance did not have to be completed in sequence. Participants could, for example, establish several communication links and then issue clearances to the aircraft.

During all training and experiment trials, the majority of the radar display was hidden from participants (see Figure 1). Only a small portion of the scope was made visible through a portal, or keyhole, which could be moved by the participant in horizontal, vertical, and diagonal directions using arrow keys on a numeric keypad. The size of the portal was approximately 1.5 inches $(3.8 \mathrm{~cm})$ square and allowed a participant to view a military aircraft traveling at maximum speed throughout the course of establishing a communication link. The participant then needed to move the portal to track the current aircraft position and to view it while issuing a clearance. Under certain Multitask ${ }^{\odot}$ simulation conditions, in order for participants to clear aircraft, they had to first find them using the portal. In this way the information acquisition aspect of the task (locating the aircraft, identifying its type, estimating its speed, etc.) was manually controlled. 


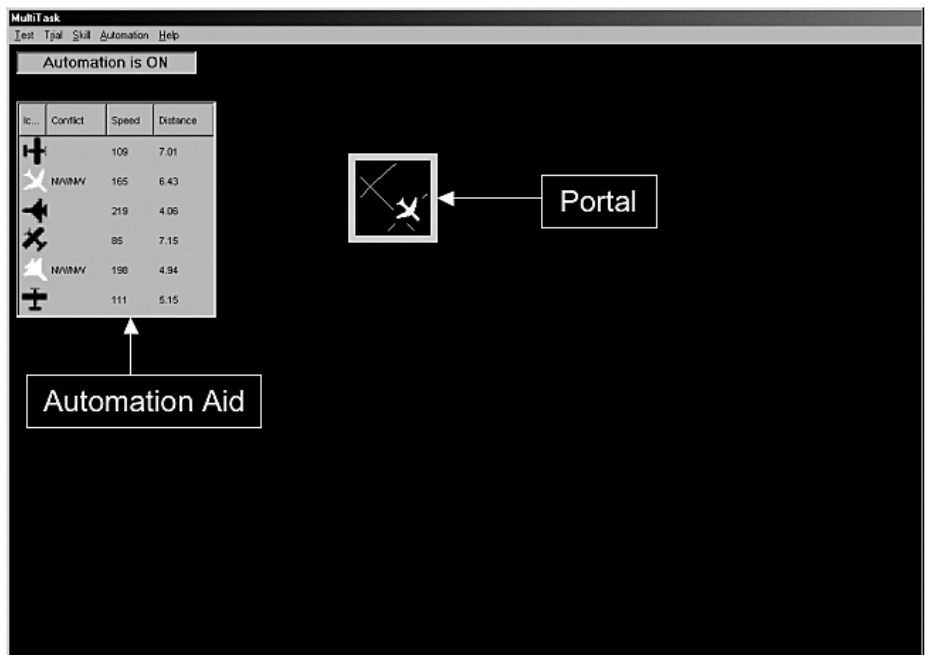

Figure 1. Multitask ${ }^{\odot}$ primary task display.

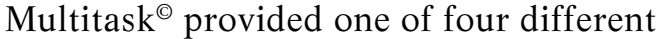
modes of automated assistance to participants, each designed to assist with a particular stage of task information processing identified in the Parasuraman et al. (2000) model, including information acquisition, information analysis, decision making, and action implementation. With respect to our labeling of the automation conditions according to these stages of information processing, Parasuraman et al. (2000) provided general descriptions of each form of automation included in their model, which we used as a guide for defining the modes of Multitask $^{\odot}$ automation. In using ATC as an example system for application of their model, Parasuraman et al. (2000) identified specific ATC technologies that they considered to be representative of each form of automation. We modeled the modes of automation in the Multitask ${ }^{\odot}$ simula- $^{-}$ tion on the basis of descriptions of the various ATC technologies in the literature (e.g., Laois \& Giannacourou, 1995; Leroux, 1993). We relate the modes of Multitask ${ }^{\odot}$ automation to these technologies in Table 1. Only one aspect of Multitask $^{\odot}$ performance was automated as part of each mode of automation. The automation was not "built up" from one mode to the next. During experiment trials, the various modes of automated assistance could be switched on or off, or adaptively applied, based on operator workload states. However, only one mode could be used per trial.
With respect to measurement of participant performance in the Multitask ${ }^{\odot}$ simulation, the number of aircraft cleared was recorded and divided by the number of aircraft presented (percentage of aircraft processed).

The secondary gauge-monitoring task presented a vertical, fixed-scale display with a moving pointer and required participants to monitor pointer movements and to detect when a deviation occurred from a central "acceptable" range on the scale (colored green) into an "unacceptable" region (colored red). The gauge display was presented on a computer monitor separate from that used to present the Multitask ${ }^{\odot}$ simulation and was positioned directly to the right of the Multitask ${ }^{\oplus}$ display. Participants were required to correct for pointer deviations (return the pointer to the acceptable range) by pressing keys on a keyboard (the up and down arrows if the pointer moved below and above the acceptable region, respectively). There were, on average, five unacceptable pointer deviations (signals) generated per minute in order for the task to constantly test an operator's ability to allocate residual attentional resources from the Multitask ${ }^{\odot}$ simulation. The number of deviations varied by \pm 1 deviation per minute. Once an unacceptable deviation occurred, the pointer remained in the red for no more than $3 \mathrm{~s}$, at which time a miss was recorded and the computer system automatically returned the pointer to the center of the display. Participants were informed, in advance of 
TABLE 1: Modes of Multitask ${ }^{\odot}$ Control (Including Manual)

Mode Description

Manual

Information acquisition

Information analysis

Decision making

Action automation
Provided no assistance. Participants manually controlled portal to find aircraft decided which aircraft to process first, and used mouse to manually execute clearances.

Provided computer-controlled movement of portal, which followed inward spiral toward center of the display. Participants could also cause portal to "lock on" aircraft when revealed through automated movement of portal (by pressing "+" or "-" keys on numeric keypad to toggle tracking on or off). Automation was considered abstraction of implementing radar tracking systems in commercial ATC operations, providing ATC with radar for tracking aircraft versus using conventional scanning radar (Parasuraman et al., 2000). No other aspect of Multitask ${ }^{\odot}$ performance (e.g., aircraft information processing, prioritizing aircraft or clearances, or issuing clearances) was automated under this mode.

Presented decision aid as part of Multitask ${ }^{\odot}$ display showing table of all aircraft currently on radarscope listed in random order. Table included aircraft type, direction of travel, speed, distance from center of display (home base), stage of clearance processing (i.e., communication link, clearance), and information on whether aircraft was involved in conflict (see Figure 1, upper left corner). Aid summarized target information for operators for decision making (prioritization of aircraft for clearances). Automation was considered similar to futuristic forms of ATC automation, including electronic data displays, trajectory projection aids, and conflict detection aids (see Laois \& Giannacourou, 1995). Mode did not provide automated control of portal motion. Participants manually directed portal to find location of aircraft or verify information presented by decision aid. Participants also manually issued clearances.

Presented decision aid showing table of all aircraft, types, and direction of travel. Decision aid sorted aircraft based on priority for clearances from top to bottom of table. Aircraft priority was defined based on likelihood of involvement in collision and distance from home base. Automation was considered to resemble clearance advisory aid in real ATC (see Laois \& Giannacourou, 1995); however, participants in study were required to effect recommendations of Multitask ${ }^{\odot}$ automation when provided. Participants were also required to manually control portal motion to search for, and locate, aircraft and to identify stage of clearance processing. Decision aid was similar to aid as part of information analysis mode but was more powerful (it prioritized aircraft for clearances). All clearances were manually implemented using mouse.

Presented feedback display with number of aircraft on radarscope and stage of processing. (Detailed icons were not presented in feedback display so as not to reveal type of aircraft.) Participants had to click on aircraft only once in order to issue clearance. Time to process aircraft was the same as in other conditions, but clearance was issued automatically after link was established. Automation was considered representative of electronic data link uploading clearances to aircraft once controller made decision. Computer assistance provided was not built up from previous modes. Participants manually controlled portal motion to locate, acquire, and integrate information on aircraft. Participants did not receive decision aids as part of information analysis and decision making modes and had to internally prioritize aircraft. Automation was applied to clearance actions subsequent to decision making. (Although computer control of portal motion could also be considered "action implementation" automation per se, it did not constitute automation relevant to response execution based on aircraft prioritization.) 
test trials, that this would happen. Monitoring performance was measured in terms of the number of unacceptable pointer deviations detected divided by the total number of deviations (the hit-to-signal ratio).

\section{Approach to Adaptive Automation}

As previously mentioned, a secondary-task approach was taken to AA (see Scerbo, 1996, for a review of methods for triggering DFAs in

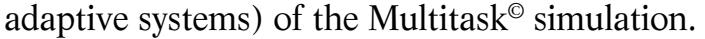
Participant performance on the gauge-monitoring task (an objective measure of workload) determined DFAs of Multitask ${ }^{\odot}$ IP functions to the human operator or to the computer.

During the final training session of the experiment, AA was applied to the Multitask ${ }^{\odot}$ simu- $^{-}$ lation and followed a cyclic control allocation schedule including 2-min periods of manual control followed by 2 -min periods of automated control. The average participant performance in the gauge-monitoring task and the standard deviation $(S D)$ for the hit-to-signal ratio were recorded. In the experiment trials, when performance of the gauge-monitoring task dropped below $1 S D$ of average task performance (for manual and automated control periods) recorded during the final training session for a particular user, the user was shifted to automated control in the Multitask ${ }^{\oplus}$ simulation. While users were under automated control, when performance of the gauge-monitoring task reached 1 $S D$ above average or was equivalent to perfect performance (a hit-to-signal ratio of 1.0), they were returned to manual control of the Multi$\operatorname{task}^{\odot}$ simulation. These criteria were defined based on pilot data (collected using 7 of the participants) suggesting that operator overload (inability to address the majority of aircraft) and underload (no aircraft conflict situations) occurred at $\pm 1 S D$ about mean performance.

\section{Experimental Design and Procedures}

The experiment followed a between-subjects design with five modes, or levels of automation (LOAs) - information acquisition, information analysis, decision making, action implementation, and completely manual control - as settings of the independent variable. Each participant performed two test trials at his or her assigned LOA. The 40 individuals not used in the pilot study were divided into five groups of 8 participants and assigned to one of the LOA conditions or the manual control condition.

The procedures for the experiment included (a) 15 min of training in Multitask ${ }^{\odot}$ under the manual control mode; (b) 15 min of training in Multitask $^{\odot}$ under the assigned LOA (participants in the manual condition received a second 15min manual training period); (c) 5 min of training in the gauge task; (d) 20 min of training in the dual-task scenario under the assigned Multitask $^{\odot}$ LOA with 2-min cycles of manual and automated control; and (e) two 20-min test trials under AA at the assigned LOA with an intervening 10-min break.

\section{DATA ANALYSIS}

Performance in the Multitask ${ }^{\odot}$ simulation and the gauge-monitoring task was recorded on a perminute basis. When transitions between control modes were indicated, they occurred at the end of a full minute during task performance.

For the statistical analyses, observations were separated into two data sets: one that included performance while in manual mode of the Multitask $^{\odot}$ simulation and another that included performance while in automated mode. For each participant, we averaged Multitask ${ }^{\odot}$ and gaugemonitoring performance across the automated minutes of a trial in order to obtain a single score for each task in each trial. Similarly, we averaged performance observations under manual minutes to obtain a single score for each task in each trial. Both the Multitask ${ }^{\odot}$ and gauge performance measures were subjected to an analysis of variance (ANOVA) with LOA as a between-subjects variable and trial as a withinsubjects variable. With respect to performance under automation, the four levels of the independent variable representing automation of the four stages of information processing were considered. In regard to the Multitask ${ }^{\odot}$ and gaugemonitoring performance measures under manual control, five levels of the LOA variable, including the completely manual control condition, were considered in the analysis.

\section{RESULTS}

\section{Primary Task Performance}

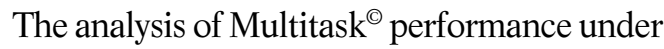


automation revealed a significant effect of trial, $F(1,25)=9.26, p<.01$, with performance in the second trial being significantly greater than performance in the first. There was also a trend in the data indicating a possible effect of LOA, which approached statistical significance, $F(3$, $27)=2.76, p=.06$; however, the result did not meet the alpha criterion of .05 .

The analysis of Multitask ${ }^{\odot}$ performance during manual minutes revealed a significant LOA effect, $F(4,33)=9.86, p<.01$, when considering the mean square error as a denominator; however, the effect was not persistent in the presence of the variance attributable to participants. An LOA by trial interaction, $F(4,33)=3.62, p<.05$, was present. According to Duncan's multiple range (MR) test, performance in the manual mode of the primary task during the second trial for those participants under the condition applying $\mathrm{AA}$ to the action implementation function was significantly better $(p<.05)$ than manual performance as part of all other AA conditions and in all other trials. In addition, the second trial of the manual control condition resulted in significantly poorer performance $(p<.05)$ than did all of the other LOA conditions and trials, with the exception of the first trial of the manual control condition and the first trial of the condition applying AA to the decision-making aspect

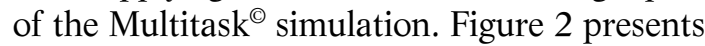
the mean Multitask ${ }^{\odot}$ performance for manual control periods as part of each AA condition and the completely manual control condition.

Because the LOA by trial interaction generally indicated that there were greater differences attributable to LOA in the second trial than in the first, we analyzed the data from the second trial separately in order to determine whether there was a main effect attributable to LOA. The analysis did reveal a significant effect of LOA, $F(4,33)=3.09, p<.05$. Duncan's MR test indicated that performance in the primary task under manual control as part of the condition applying AA to the action implementation function was significantly better than performance under manual control as part of all other AA conditions and the completely manual control condition.

It may have been possible that the second trial

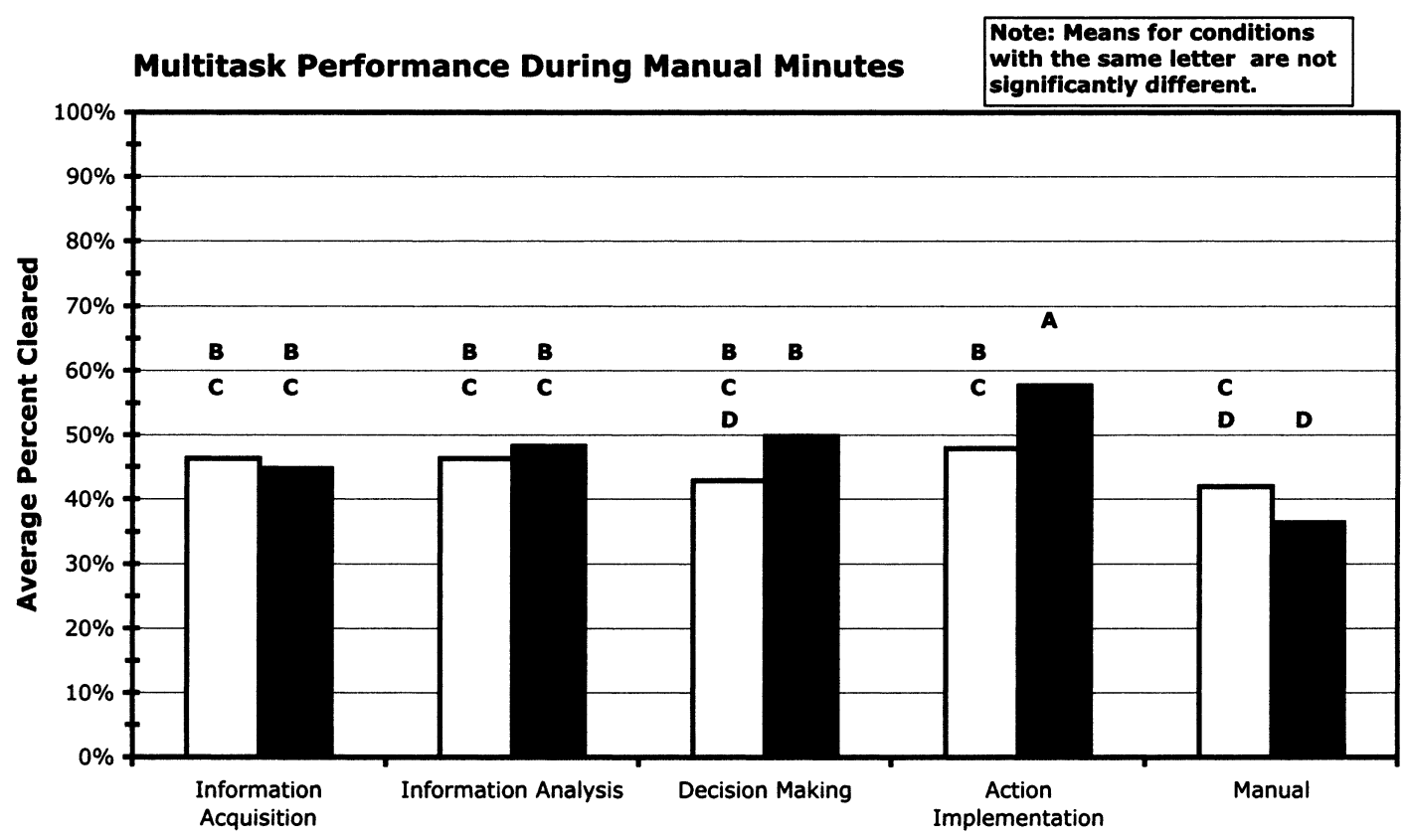

Level of Automation

\section{口Trial 1 Trial 2}

Figure 2. Primary task performance during manual minutes by LOA and trial. 
was more sensitive for revealing performance differences attributable to the LOA, as operators had the experience of the first test trial to refine their control strategies, which allowed them to better exploit the features of the AA as applied to the various IP functions. In general, Figure 2 suggests that there was a trend for better manual control performance as part of the AA conditions, as compared with the manual control condition.

\section{Secondary Task Performance}

The analysis of performance on the secondary, gauge-monitoring task during automation of the Multitask $^{\odot}$ simulation indicated a significant effect attributable to $\operatorname{LOA}, F(3,27)=3.43, p<$ .05 . Duncan's MR test revealed that the hit-tosignal ratio in the secondary task was significantly higher (workload was lower) when AA was applied to the information acquisition and action implementation functions of the primary task than when Multitask ${ }^{\odot}$ information analysis was automated $(p<.05)$. Figure 3 presents a plot of the mean signal detection rates in the secondary task during automated and manual control periods as part of the Multitask ${ }^{\odot}$ AA conditions. The plot reflects the impact of the AA, as applied to the various IP functions, on operator residual attentional resources. The decision aids provided as part of the information analysis and decisionmaking conditions included additional visual displays (as compared with automation of information acquisition and action implementation) that may have increased both visual attention and cognitive-processing loads for operators, leading to poorer gauge performance under automation of those conditions.

During the manual minutes, there was no significant effect of LOA, although there was a highly significant effect of trial, $F(1,33)=$ $12.36, p<.01$. Post hoc analysis revealed gaugemonitoring task performance (objective workload) to be significantly worse $(p<.05)$ during the second trial than during the first. It is possible that operators developed a better understanding of the automation modes during the first trial and, consequently, paid more attention to the computer assistance in the second trial.

\section{Gauge Task Performance}

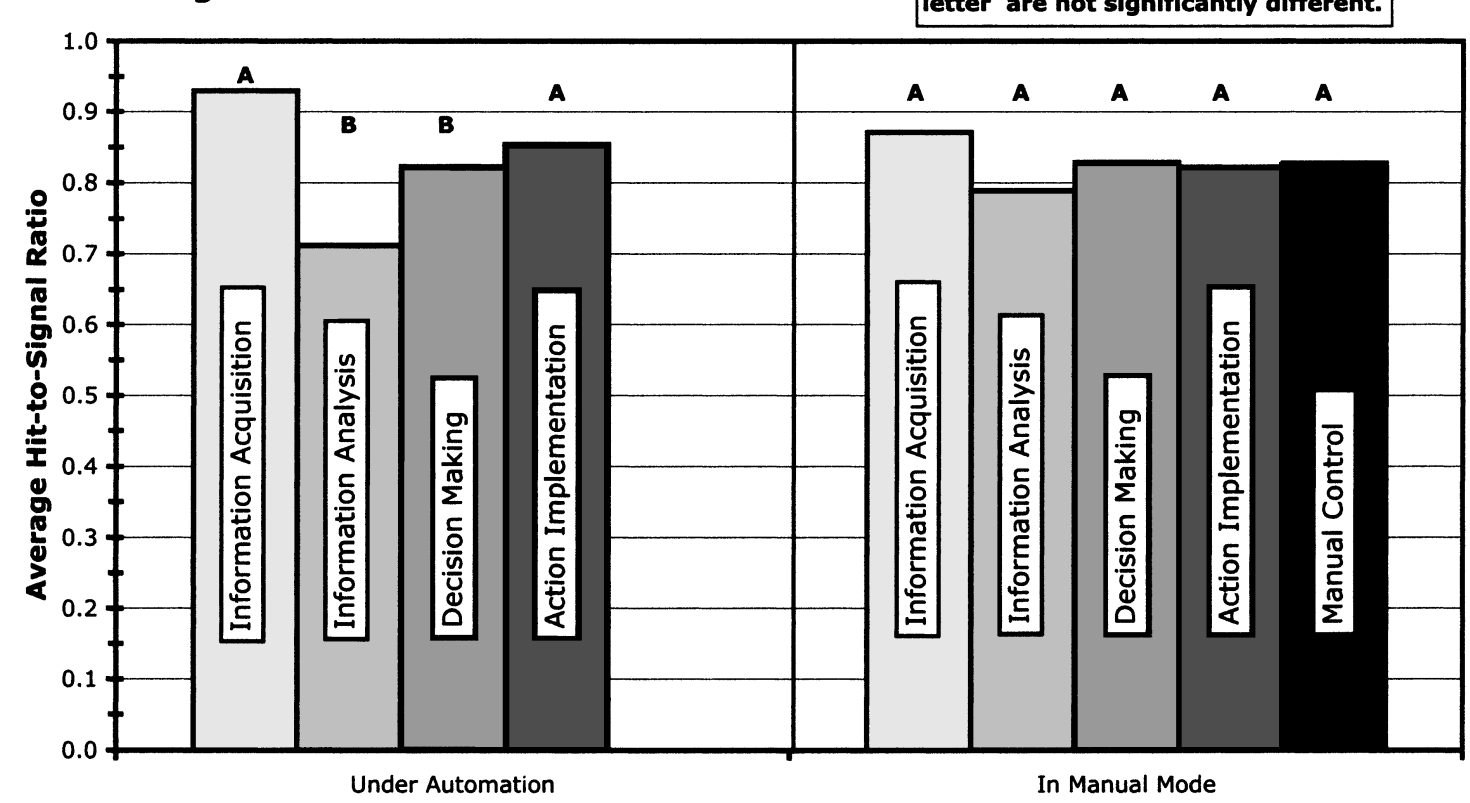

Level of Automation

Figure 3. Gauge task (secondary task) performance by LOA under automated and manual control. 


\section{DISCUSSION}

These results provide evidence that the effectiveness of $\mathrm{AA}$ is dependent on the stage of human-machine system information processing, which is flexibly automated, and that AA systems can be sufficiently complex so as to require a significant amount of training. (These are potentially important human factors design considerations for adaptive systems.) It was our expectation that humans would be better able to adapt to AA of lower order IP functions, and the function of action implementation appears to be well suited to AA. This observation is in line with the findings of historical AA research (e.g., Hilburn et al., 1993; Parasuraman et al., 1993). Our hypothesis that primary task performance would be greatest when automation was applied to the action implementation aspect of the task was also supported. We expected the disruption and costs to task performance caused by control mode switching (as part of AA) to be greater when operators were using decision aids versus aids for perceptuomotor functions, such as action implementation, and that it would be more difficult for operators to return to manual control under AA of information analysis or decision making because of the degree of cognitive complexity of the automation aid. The finding that manual control performance as part of AA of action implementation was better than under the other AA conditions and the completely manual control condition most directly supports this expectation.

It is possible that under specific task circumstances, AA can benefit all four stages of information processing, as compared with traditional (static) function allocation. However, our findings support the notion that humans are better at adapting to that which is transparent to them. It is much easier to form a mental model of a system performing automated aircraft handoffs for a controller, or helping a radar operator find targets, than it is to do so for a much more opaque function, such as trying to help the operator make decisions on aircraft clearances. In the former situations, the effect of automation either does or doesn't occur and the operator can clearly assess the reliability. In the latter situation, additional cognitive processing is required to validate the system function against the operator's mental model. It is important here to not confuse the "transparency" of the implications of the various automated functions on system performance (i.e., how easily an operator can comprehend whether the function worked) with the transparency or visibility of automated system functions through an interface. It is very possible that even if operators are provided feedback on automation states through information displays, they may not be able to quickly and accurately comprehend whether the automation worked or is accurate in its advice, as a result of the complexity of the information content and the associated cognitive processing. This again goes back to the cognitive complexity of the particular type of aid - that is, whether the automation is performing simple perceptuomotor assistance or whether it is making complex decisions or judgments that require mental simulation, calculation, and so forth, for operators to validate.

It is also possible that performance decrements under the AA conditions, in general, might have resulted from a lack of warnings of automation state changes for operators during the experimental trials. Unfortunately, the lack of warnings of control mode switching does not provide insight into why differences in performance might have occurred among the various AA conditions. Although sensory cuing of automation state changes in adaptive systems has been the topic of some contemporary AA research, and results have revealed potential benefits of modal and bimodal cuing (Kaber \& Wright, 2003), it was not the focus of this study. It may be worthwhile for future research to consider interaction effects of AA applied to various IP functions and types of sensory warnings of automation state changes on operator performance.

Beyond these findings and explanations, in comparing performance under the manual control condition with both automated and manual control periods as part of all AA conditions, with the exception of the first trial applying AA to the decision-making function, we found that performance was always better under AA. This finding supports our hypothesis that AA of various IP functions would be superior to manual control. There was no static automation condition included in this study, as a basis for comparison with the various forms of AA, because several prior investigations have already demonstrated 
the benefits of AA relative to traditional automation (e.g., Parasuraman et al., 1993; Scallen, Hancock, \& Duley, 1995). The focus of this research was on identifying the differential effects of the various forms of AA in the ATC simulation. We concentrated on comparisons of manual control performance, as part of AA trials, with performance on a completely manual control condition in order to assess the return-to-manual deficit hypothesis. Taken together, the primary task performance results under automated and manual control periods also support our speculation that the higher order IP functions would show less benefit of AA as compared with lowerorder functions (information acquisition and action implementation).

Considering the results on secondary task performance, or the workload measure, signal detection performance was greater under both automated and manual minutes when AA was applied to the information acquisition aspect of the task, particularly when compared with automated control as part of AA of the information analysis function. As we hypothesized, the automation under the information acquisition condition appeared to relieve some of the physical task workload and time pressure for participants, as the computer automatically controlled the motion of the portal in searching for aircraft and allowed participants to attend to the secondary task. In contrast, automation of information analysis appeared to reduce the time available to attend to the secondary task. It is possible that the decision aid display as part of this condition held the operators' attention in their attempts to effect an optimal processing strategy. Wiener (1988) and Parasuraman et al. (2000) have referred to automation that results in increased operator workload as "clumsy" automation. In general, the complexity of our information analysis automation and the visual attention required by the displays may have caused an increase in primary task workload. However, this may have also been influenced by operator familiarity with the automation and individual differences (Riley \& Parasuraman, 1997).

\section{CONCLUSIONS AND FUTURE RESEARCH}

Up to this point in time, no work has made direct comparison of the ability of humans to deal with, and exploit, AA of low-order IP functions versus higher cognitive functions. We presented an experiment that examined the relative effects of AA applied to the human-machine system IP functions of information acquisition, information analysis, decision making, and action implementation, as defined in one model of types and levels of automation. In general, the study demonstrated the effectiveness of AA to be dependent on the stage of task performance that is flexibly automated; specifically, humans may be better able to adapt to AA applied to lower order sensory and psychomotor functions, such as information acquisition and action implementation, as compared with AA applied to cognitive (analysis and decision making) tasks. Our results also provide support for the use of AA, as compared with completely manual control.

In general, a more systematic approach needs to be taken to AA research, including consideration of the theoretical frameworks and models of automation that have been proposed as bases for formulating novel research hypotheses and designing new empirical studies of the implications of implementing AA. Integration of the results presented here and those of future systematic experiments within the frameworks of automation presented in the literature may ultimately serve as a design rationale for the development of future adaptive systems.

Related to the findings of this work, other recent AA research (Kaber \& Endsley, 2004) has demonstrated that the form of complex system automation and the specific approach to DFAs significantly influence not only operator performance but SA as well. Similar to our study, Kaber and Endsley (2004) found that lower levels of automation, like action implementation aiding, promote performance when adaptively applied to complex systems but also have a negative effect on SA. This should be of concern to designers of automated systems because it may mean that applying AA to psychomotor functions in order to increase performance in the near term may undermine SA over extended periods. Future research should also involve comparison of various approaches to triggering DFAs, as part of AA, in terms of operator performance and SA.

\section{ACKNOWLEDGMENTS}

This work was completed while Melanie 
Wright and Michael Clamann worked as a research associate and research assistant, respectively, at North Carolina State University. The research was supported by a grant from NASA Langley Research Center, Psychological/Physiological Stressors and Factors Program, Hampton, Virginia (Grant No. NAG-1-01039). Lance Prinzel was the technical monitor. The views and conclusions presented in this paper are those of the authors and do not necessarily reflect the views of NASA.

\section{REFERENCES}

Atwood, M. E., \& Polson, P. G. (1976). A process model for water jug problems. Cognitive Psychology, 8, 191-216.

Endsley, M. R., \& Kaber, D. B. (1999). Level of automation effects on performance, situation awareness and workload in a dynamic control task. Ergonomics, 42, 462-492.

Hadley, G. A., Prinzel, L. J., Freeman, F. G., \& Mikulka, P. J. (1999). Behavioral, subjective and psychophysiological correlates of various schedules of short-cycle automation. In M. W. Scerbo \& M. Mouloua (Eds.), Automation technology and human performance (pp. 139-143). Mahwah, NJ: Erlbaum.

Hilburn, B., Jorna, P., Byrne, E. A., \& Parasuraman, R. (1997). The effect of adaptive air traffic control (ATC) decision aiding on controller mental workload. In M. Mouloua \& J. M. Koonce (Eds.), Human-automation interaction: Research and practice (pp. 84-91). Mahwah, NJ: Erlbaum.

Hilburn, B., Molloy, R., Wong, D., \& Parasuraman, R. (1993). Operator versus computer control of adaptive automation. In R. S. Jensen \& D. Neumeister (Eds.), Proceedings of the 7th International Symposium on Aviation Psychology (pp. 161-166) Columbus: Ohio State University, Department of Aviation.

Kaber, D. B., Clamann, M., Labiad, N., \& Wright, M. C. (2001) MultitaskIA ${ }^{\odot}$ (Version 2.0) [Computer software]. Raleigh: North Carolina State University, Department of Industrial Engineering.

Kaber, D. B., \& Endsley, M. R. (1997). The combined effect of level of automation and adaptive automation on human performance with complex, dynamic control systems. In Proceedings of the Human Factors and Ergonomics Society 41st Annual Meeting (pp. 205-209). Santa Monica, CA: Human Factors and Ergonomics Society.

Kaber, D. B., \& Endsley, M. R. (2004). The effects of level of automation and adaptive automation on human performance, situation awareness and workload in a dynamic control task Theoretical Issues in Ergonomics Science, 5, 113-153.

Kaber, D. B., \& Riley, J. (1999). Adaptive automation of a dynamic control task based on secondary task workload measurement. International Journal of Cognitive Ergonomics, 3, 169-187.

Kaber, D. B., Riley, J., Tan, K., \& Endsley, M. R. (2001). On the design of adaptive automation for complex systems. International Journal of Cognitive Ergonomics, 5, 37-57.

Kaber, D. B., \& Wright, M. C. (2003, August). Automation-state changes and sensory cueing in telerobot control. In Proceedings of the XVth Triennial Congress of the International Ergonomics Association [CD-ROM] (Vol. 4). Seoul: Ergonomics Society of Korea.

Laois, L., \& Giannacourou, M. (1995). Perceived effects of advanced ATC functions on human activities: Results of a survey on controllers and experts. In Proceedings of the Eighth International Symposium on Aviation Psychology (pp. 392-397). Columbus: Ohio State University.

Leroux, M. (1993). The role of expert systems in future cooperative tools for air traffic controllers. In Proceedings of the Seventh International Symposium on Aviation Psychology (pp. 335-340). Columbus: Ohio State University.

Parasuraman, R. (1987). Human-computer monitoring. Human Factors, 29, 695-706.
Parasuraman, R. (2000). Designing automation for human use: Empirical studies and quantitative models. Ergonomics, 43, 931-951.

Parasuraman, R., Mouloua, M., \& Molloy, R. (1996). Effects of adaptive task allocation on monitoring of automated systems. Human Factors, 38, 665-679.

Parasuraman, R., Mouloua, M., Molloy, R., \& Hilburn, B. (1993). Adaptive function allocation reduces performance costs of static automation. In R. S. Jensen \& D. Neumeister (Eds.), Proceedings of the Seventh International Symposium on Aviation Psychology (pp. 178-185). Columbus: Ohio State University, Department of Aviation.

Parasuraman, R., Wickens, C. D., \& Sheridan, T. (2000). A model for types and levels of human interaction with automation. IEEE Transactions on Systems, Man, and Cybernetics, 30, 286-297.

Pervin, L. A. (1984). Personality. New York: Wiley.

Rauterberg, M. (1992). A method of quantitative measurement of cognitive complexity. In G. van der Veer, M. Tauber, S. Bagnara, \& M. Antalovits (Eds.), Human-computer interaction: Tasks and organisation (pp. 295-307). Rome, Italy: CUD.

Riley, V., \& Parasuraman, R. (1997). Humans and automation: Use, misuse, disuse, abuse. Human Factors, 39, 230-253.

Rouse, W. B. (1977). Human-computer interaction in multi-task situations. IEEE Transactions on Systems, Man and Cybernetics, SMC-7, 384-392.

Scallen, S. F., Hancock, P. A., \& Duley, J. A. (1995). Pilot performance and preference for short cycles of automation in adaptive function allocation. Applied Ergonomics, 26, 397-403.

Scerbo, M. W. (1996). Theoretical perspectives on adaptive automation. In R. Parasuraman \& M. Mouloua (Eds.), Human performance in automated systems: Theory and applications (pp. 37-64). Mahwah, NJ: Erlbaum.

Sheridan, T. B., \& Verplanck, W. L. (1978). Human and computer control of undersea teleoperators. Cambridge: Massachusetts Institute of Technology, Man-Machine Laboratory.

Wickens, C. D. (1992). Engineering psychology and human performance (2nd ed.). New York: HarperCollins.

Wickens, C. D., Mavor, A., Parasuraman, R., \& McGee, J. (1998). The future of air traffic control: Human operators and automation. Washington, DC: National Academy Press.

Wiener, E. L. (1988). Cockpit automation. In E. L. Wiener \& D. C. Nagel (Eds.), Human factors in aviation (pp. 433-459). San Diego, CA: Academic Press.

David B. Kaber is an associate professor of industrial engineering in the Edward P. Fitts Department of Industrial Engineering at North Carolina State University. He received his Ph.D. in industrial engineering from Texas Tech University in 1996.

Melanie C. Wright is an assistant professor at Duke University Medical Center. She received her Ph.D. in industrial engineering from North Carolina State University in 2002.

Lawrence J. Prinzel III is an aerospace technologist and research psychologist at NASA Langley Research Center, a project manager under the NASA Airspace Systems Program, and a human factors researcher under the Aviation Safety Program. He received his $\mathrm{Ph}$.D. from Old Dominion University in engineering and systems psychology in 1997.

Michael P. Clamann is a human factors consultant with Micro Analysis \& Design, Inc., Boulder Colorado. He received his master's degree in psychology at North Carolina State University in 2002.

Date received: October 28, 2003

Date accepted: November 11, 2004 